\title{
A NEW SUMS AND ITS RECIPROCITY THEOREM
}

\author{
GUOHUI CHEN AND DI HAN
}

Received 10 March, 2015

\begin{abstract}
The main purpose of this paper is introduced a new sums analogous to Dedekind sums, then using the analytic method and the properties of Dirichlet $L$-functions to study the arithmetical properties of this sums, and give an interesting reciprocity theorem for it.
\end{abstract}

2010 Mathematics Subject Classification: 11M20

Keywords: a new sums, Dedekind sums, reciprocity theorem, identity, analytic method

\section{INTRODUCTION}

For a positive integer $k$ and an arbitrary integer $h$, the classical Dedekind sums $S(h, k)$ is defined by

$$
S(h, k)=\sum_{a=1}^{k}\left(\left(\frac{a}{k}\right)\right)\left(\left(\frac{a h}{k}\right)\right),
$$

where

$$
((x))= \begin{cases}x-[x]-\frac{1}{2} & \text { if } x \text { is not an integer } \\ 0 & \text { if } x \text { is an integer. }\end{cases}
$$

The various arithmetical properties of $S(h, k)$ were investigated by many authors, one of the most important results (see Tom M. Apostol [2] or L. Carlitz [3]) is its reciprocity theorem. That is, for all positive integers $h$ and $q$ with $(h, q)=1$, we have the identity

$$
S(h, q)+S(q, h)=\frac{h^{2}+q^{2}+1}{12 h q}-\frac{1}{4} .
$$

The other properties of $S(h, q)$ can also be found in [2], [3], [4], [6], [7] [8] and [9]. But we think that the formula (1.1) is very important and interesting, because from it we can compute the value of $S(h, q)$ by $S(q, h)$, providing $(h, q)=1$.

This work is supported by the N. S. F. $(11371291,11471258)$ of P. R. China and G.I.C.F. (YZZ14004) of Northwest University. 
In this paper, we introduce a new sums $C(h, q)$ as follows:

$$
C(h, q)=\sum_{a=1}^{q} \cot \left(\frac{\pi h a}{q}\right) \cot \left(\frac{\pi a}{q}\right),
$$

where $\sum^{\prime}$ denotes the summation over all $a$ such that $(a, q)=1,(h, q)=1$ and $\cot (x)=\cos (x) / \sin (x)$. We also provide $C(h, q)=0$, if $q \mid h$.

This sums looks very similar to Dedekind sums, so we think that it must have some similar properties with Dedekind sums. Based on this reason, we use the analytic method and the properties of Dirichlet $L$-functions to study the reciprocity properties of $C(h, k)$, and obtain an interesting reciprocity theorem. That is, we shall prove the following:

Theorem 1. For any integers $h>1$ and $q>1$ with $(h, q)=1$, we have the reciprocity formula

$$
\frac{1}{q} \sum_{d \mid q} C(h, d)+\frac{1}{h} \sum_{d \mid h} C(q, d)=\frac{q^{2}+h^{2}+1}{3 q h}-1,
$$

where $\sum_{d \mid q}$ denotes the summation over all divisors $d$ of $q$.

Theorem 2. For any square-full number q, we have the identity

$$
\sum_{a=1}^{q} \sum_{b=1}^{q} K(a, 1 ; q) K(b, 1 ; q) C(a \bar{b}, q)=\frac{1}{3} \cdot q^{2} \cdot \phi^{2}(q) \cdot \prod_{p \mid q}\left(1+\frac{1}{p}\right),
$$

where $K(m, n ; q)=\sum_{u=1}^{q} e\left(\frac{m u+n \bar{u}}{q}\right)$ is the Kloostermann sums, $e(y)=e^{2 \pi i y}, \prod_{p \mid q}$ denotes the product over all distinct prime divisors $p$ of $q$, and $u \cdot \bar{u} \equiv 1 \bmod q$.

Note that $C(h, 1)=C(q, 1)=0$, so from our Theorem 1 we may immediately deduce the following:

Corollary 1. For any odd primes $p$ and $q$ with $p \neq q$, we have the reciprocity formula

$$
\frac{C(p, q)}{q}+\frac{C(q, p)}{p}=\frac{p^{2}+q^{2}+1}{3 p q}-1
$$

\section{SOME LEMMAS}

In this section, we shall give some lemmas which are necessary in the proof of our theorems. First we have the following: 
Lemma 1. Let $q>2$ be an integer, and let $\chi$ be any Dirichlet character $\bmod q$ with $\chi(-1)=-1$. Then we have the identity

$$
L(1, \chi)=\frac{\pi}{2 q} \sum_{r=1}^{q} \chi(r) \cot \left(\frac{\pi r}{q}\right),
$$

where $L(1, \chi)$ denotes Dirichlet $L$-function corresponding to $\chi$ mod $q$.

Lemma 2. Let $q \geq 3$ be an integer, then for any integer $h$ with $(h, q)=1$, we have the identity

$$
S(h, q)=\frac{1}{\pi^{2} q} \sum_{d \mid q} \frac{d^{2}}{\phi(d)} \sum_{\substack{\chi \bmod d \\ \chi(-1)=-1}} \bar{\chi}(h)|L(1, \chi)|^{2},
$$

where $\chi$ runs through the Dirichlet characters $\bmod d$ with $\chi(-1)=-1$.

Proof. The proofs of Lemma 1 and Lemma 2 can be found in [8].

Lemma 3. Let $q \geq 3$ be an integer, then for any integer $h$ with $(h, q)=1$, we have the identity

$$
C(h, q)=\frac{4 q^{2}}{\pi^{2} \phi(q)} \sum_{\substack{\chi \bmod q \\ \chi(-1)=-1}} \chi(h)|L(1, \chi)|^{2} .
$$

Proof. For any integer $a$ with $(a, q)=1$, note that $\sum_{b=1}^{q} \chi(b) \cot \left(\frac{\pi b}{q}\right)=0$ if $\chi(-1)=1$, from Lemma 1 and the orthogonality of characters $\bmod q$ we have

$$
\begin{aligned}
\sum_{\substack{\chi \bmod q \\
\chi(-1)=-1}} \bar{\chi}(a) L(1, \chi) & =\sum_{\substack{\chi \bmod q \\
\chi(-1)=-1}} \bar{\chi}(a)\left(\frac{\pi}{2 q} \sum_{r=1}^{q} \chi(r) \cot \left(\frac{\pi r}{q}\right)\right) \\
& =\frac{\pi}{2 q} \sum_{\chi \bmod q} \bar{\chi}(a)\left(\sum_{r=1}^{q} \chi(r) \cot \left(\frac{\pi r}{q}\right)\right)=\frac{\pi \phi(q)}{2 q} \cdot \cot \left(\frac{\pi a}{q}\right) .
\end{aligned}
$$

or

$$
\cot \left(\frac{\pi a}{q}\right)=\frac{2 q}{\pi \phi(q)} \sum_{\substack{\chi \bmod q \\ \chi(-1)=-1}} \bar{\chi}(a) L(1, \chi) .
$$

Then from the definition of $C(h, q),(2.1)$ and the orthogonality of characters $\bmod q$ we have the identity

$$
C(h, q)=\sum_{a=1}^{q} \cot \left(\frac{\pi h a}{q}\right) \cot \left(\frac{\pi a}{q}\right)
$$




$$
\begin{aligned}
& =\frac{4 q^{2}}{\pi^{2} \phi^{2}(q)} \sum_{\substack{\chi_{1} \bmod q \\
\chi_{1}(-1)=-1}} \sum_{\substack{\chi_{2} \bmod q \\
\chi_{2}(-1)=-1}} \sum_{a=1}^{q} \bar{\chi}_{1}(h a) \bar{\chi}_{2}(a) L\left(1, \chi_{1}\right) L\left(1, \chi_{2}\right) \\
& =\frac{4 q^{2}}{\pi^{2} \phi(q)} \sum_{\substack{\chi \bmod q \\
\chi(-1)=-1}} \bar{\chi}(h)|L(1, \chi)|^{2}=\frac{4 q^{2}}{\pi^{2} \phi(q)} \sum_{\substack{\chi \bmod q \\
\chi(-1)=-1}} \chi(h)|L(1, \chi)|^{2} .
\end{aligned}
$$

This proves Lemma 3.

Lemma 4. Let $q \geq 3$ be a square-full number. Then we have the identity

$$
\sum_{\substack{\chi \bmod q \\ \chi(-1)=-1}}^{*}|L(1, \chi)|^{2}=\frac{\pi^{2}}{12} \frac{\phi^{3}(q)}{q^{2}} \prod_{p \mid q}\left(1+\frac{1}{p}\right),
$$

where $\sum_{\substack{\chi \bmod q \\ \chi(-1)=-1}}^{*}$ denotes the summation over all primitive odd characters $\bmod q$.

Proof. The proof of Lemma 4 can be found in [8].

Lemma 5. Let $q$ be a square-full number. Then for any non-primitive character $\chi \bmod q$, we have the identity

$$
\tau(\chi)=\sum_{a=1}^{q} \chi(a) e\left(\frac{a}{q}\right)=0 .
$$

Proof. It is clear that Gauss sums $|\tau(\chi)|$ is a multiplicative function of $\bmod q$ (see references [1] and [5]), so without loss of generality, we can assume $q=p^{\alpha}$, where $p$ be a prime and $\alpha \geq 2$. Now if $\chi$ is a non-primitive character $\bmod p^{\alpha}$, then it is also a character mod $p^{\alpha-1}$. So from the properties of the trigonometric sums we know that for any positive integers $q \geq 2$ and integer $n$ with $(n, q)=1$, we have the identity

$$
\sum_{u=0}^{q-1} e\left(\frac{u n}{q}\right)=0
$$

From (2.2) and the definition of the reduce residue system modulo $p^{\alpha}$ we have

$$
\begin{gathered}
\tau(\chi)=\sum_{a=1}^{p^{\alpha}} \chi(a) e\left(\frac{a}{p^{\alpha}}\right)=\sum_{u=0}^{p-1} \sum_{v=1}^{p^{\alpha-1}} \chi\left(u p^{\alpha-1}+v\right) e\left(\frac{u p^{\alpha-1}+v}{p^{\alpha}}\right) \\
=\sum_{u=0}^{p-1} \sum_{v=1}^{p^{\alpha-1}} \chi(v) e\left(\frac{u}{p}+\frac{v}{p^{\alpha}}\right)=\sum_{v=1}^{p^{\alpha-1}} \chi(v) e\left(\frac{v}{p^{\alpha}}\right) \sum_{u=0}^{p-1} e\left(\frac{u}{p}\right)=0 .
\end{gathered}
$$

This proves Lemma 5 . 


\section{Proof of Theorems}

In this section, we shall complete the proof of our theorems.

Proof of Theorem 1. For any positive integers $h>1$ and $q>1$ with $(h, q)=1$, note that $C(h, 1)=0$ and $(h, d)=1$ for all $d \mid q$. So from Lemma 3 and Lemma 2 we have

$$
\frac{1}{q} \sum_{d \mid q} C(h, d)=\frac{4}{\pi^{2} q} \sum_{d \mid q} \frac{d^{2}}{\phi(d)} \sum_{\substack{\chi \bmod d \\ \chi(-1)=-1}} \chi(h)|L(1, \chi)|^{2}=4 S(h, q) .
$$

Similarly, using the method of proving (3.1) we can also deduce that

$$
\frac{1}{h} \sum_{d \mid h} C(q, d)=\frac{4}{\pi^{2} h} \sum_{d \mid h} \frac{d^{2}}{\phi(d)} \sum_{\substack{\chi \bmod d \\ \chi(-1)=-1}} \chi(q)|L(1, \chi)|^{2}=4 S(q, h) .
$$

Combining (1.1), (3.1) and (3.2) we may immediately deduce the reciprocity formula

$$
\frac{1}{q} \sum_{d \mid q} C(h, d)+\frac{1}{h} \sum_{d \mid h} C(q, d)=S(h, q)+S(q, h)=\frac{q^{2}+h^{2}+1}{3 q h}-1 .
$$

This proves Theorem 1 .

Proof of Theorem 2. If $q$ is a square-full number and $\chi$ is not a primitive character mod $q$, then from Lemma 5 we know that $\tau(\chi)=0$. If $\chi$ is a primitive character $\bmod q$, then $|\tau(\chi)|^{2}=q$. Note that the identities $\tau^{2}(\bar{\chi})=\overline{\tau(\chi)}^{2}$ and

$\sum_{a=1}^{q} \chi(a) K(a, 1 ; q)=\sum_{u=1}^{q} \sum_{a=1}^{q} \chi(a) e\left(\frac{a u+\bar{u}}{q}\right)=\tau(\chi) \cdot \sum_{u=1}^{q} \bar{\chi}(u) e\left(\frac{\bar{u}}{q}\right)=\tau^{2}(\chi)$,

from Lemma 3 and Lemma 4 we have

$$
\begin{aligned}
& \sum_{a=1}^{q} \sum_{b=1}^{q} K(a, 1 ; q) K(b, 1 ; q) C(a \bar{b}, q) \\
& =\frac{4 q^{2}}{\pi^{2} \phi(q)} \sum_{\substack{\chi \bmod q \\
\chi(-1)=-1}} \sum_{a=1}^{q} \chi(a) K(a, 1 ; q) \sum_{b=1}^{q} \bar{\chi}(b) K(b, 1 ; q)|L(1, \chi)|^{2} \\
& =\frac{4 q^{2}}{\pi^{2} \phi(q)} \sum_{\substack{\chi \bmod q \\
\chi(-1)=-1}} \tau^{2}(\chi) \cdot \tau^{2}(\bar{\chi}) \cdot|L(1, \chi)|^{2}
\end{aligned}
$$




$$
\begin{aligned}
& =\frac{4 q^{2}}{\pi^{2} \phi(q)} \sum_{\substack{\chi \bmod q \\
\chi(-1)=-1}}^{*}|\tau(\chi)|^{4} \cdot|L(1, \chi)|^{2} \\
& =\frac{4 q^{4}}{\pi^{2} \phi(q)} \cdot \frac{\pi^{2}}{12} \frac{\phi^{3}(q)}{q^{2}} \prod_{p \mid q}\left(1+\frac{1}{p}\right)=\frac{1}{3} \cdot q^{2} \cdot \phi^{2}(q) \cdot \prod_{p \mid q}\left(1+\frac{1}{p}\right) .
\end{aligned}
$$

This completes the proof of Theorem 2.

\section{ACKNOWLEDGEMENT}

The authors would like to thank the referees for their very helpful and detailed comments, which have significantly improved the presentation of this paper.

\section{REFERENCES}

[1] T. M. Apostol, Introduction to Analytic Number Theory. New York: Springer-Verlag, 1976.

[2] T. M. Apostol, Modular Functions and Dirichlet Series in Number Theory. New York: SpringerVerlag, 1976.

[3] L. Carlitz, "The reciprocity theorem of Dedekind sums," Pacific J. of Math., vol. 3, pp. 513-522, 1953.

[4] J. B. Conrey, E. Fransen, R. Klein, and C. Scott, "Mean values of Dedekind sums," Journal of Number Theory, vol. 56, pp. 214-226, 1996, doi: 10.1006/jnth.1996.0014.

[5] K. Ireland and M. Rosen, A classical introduction to modern number theory. New York: SpringerVerlag, 1982.

[6] C. Jia, "On the mean value of Dedekind sums," Journal of Number Theory, vol. 87, pp. 173-188, 2001, doi: 10.1006/jnth.2000.2580.

[7] Y. Liu and W. Zhang, "A hybrid mean value related to the Dedekind sums and Kloosterman sums," Acta Mathematica Sinica, vol. 27, pp. 435-440, 2011, doi: 10.1007/s10114-010-9192-2.

[8] W. Zhang, "On the mean values of Dedekind sums," Journal de Theorie des Nombres, vol. 8, pp. 429-442, 1996.

[9] W. Zhang, "A note on the mean square value of the Dedekind sums," Acta Mathematica Hungarica, vol. 86, pp. 275-289, 2000, doi: 10.1023/A:1006724724840.

Authors' addresses

\section{Guohui Chen}

College of Mathematics and Statistics, Hainan Normal University, Hainan, P.R.China

E-mail address: 3478823460 qq.com

\section{Di Han}

School of Mathematics, Northwest University, Xi'an, Shaanxi, P. R. China

E-mail address: handi5150163.com 